\title{
Advances of Magnetic Memory Testing Technology
}

\author{
Shujun $\mathrm{Liu}^{1}{ }^{1} \mathrm{a}^{*}$, Shenglin $\mathrm{Li}^{1}$, Ming Jiang ${ }^{1}$, Dean $\mathrm{He}^{1}$ \\ 'Logistics Engineering University, Chongqing 401311, China \\ a119042856@qq.com
}

Keywords: Magnetic memory testing, NDT, Stress concentration, Defect, Quantitative identification

\begin{abstract}
The Magnetic Memory Testing Technology is a new type of NDT. It is an effective method for the detection and evaluation of oil and gas pipelines, and it has a good application prospect. This paper reviews the development course of magnetic memory testing technology, summarizes the Magnetic Memory Testing in the micro mechanism, signal processing, magnetic memory testing criterion, the stress concentration area of magnetic memory testing stress concentration or defect quantitative identification, magnetic memory testing instrument research and development and other aspects of the research achievements, analyzes the problem of the Magnetic Memory Testing, and points out the development trend of the Magnetic Memory Testing.
\end{abstract}

\section{Introduction}

With the rapid development of pipeline transportation industry, the maintenance and management of the pipeline, the leakage prevention and the safe operation of the pipeline have been paid more attention to. Once the pipeline accident, it will not only drain a lot of energy, causing huge economic losses, but also cause serious consequences of social life and living environment. Therefore, how to accurately grasp the status of the pipeline, nip in the bud, has become a major issue of common concern at home and abroad. The traditional Nondestructive testing methods, such as magnetic flux leakage, magnetic powder, ultrasonic and eddy current, can only find most of micro defects and the macro defect which has been formed, but cannot find the development of micro forming defects and stress concentration in these regions. While in fact, those are the danger zone of pipeline failure. Therefore, it is very necessary to detect the early damage or hidden damage, especially the stress concentration in the pipeline.

\section{The Proposal of Magnetic Memory Testing}

In late 1990s, Russian scholar Doubov first put forward a new nondestructive testing technology Metal Magnetic Memory Testing, referred to as magnetic memory testing. As shown in Fig. 1, its principle can be expressed as: For the effect of the load, the domain organization orientation will cause magnetostriction properties and irreversible reorientation in the Ferromagnetic components working in a geomagnetic field. The maximum leakage magnetic field is formed in the stress and deformation zone. The tangential component of the magnetic field in the stress concentration area $H_{P}(x)$ has the maximum value, while the sign of the normal component $H_{P}(y)$ changes and has zero-crossing position. The irreversible change of the magnetic state remains after the elimination of the working load. By the testing of the magnetic flux leakage field's normal component $H_{P}(y)$ and the Calculation of gradient, the stress concentration position of the component can be exactly deduced $[1,2]$. 


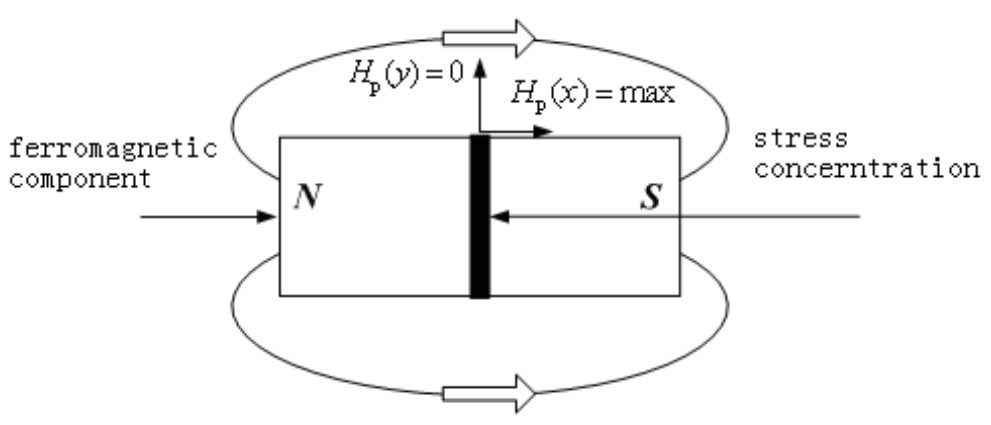

Fig. 1 Schematic of MMT.

Magnetic Memory Testing technology is the only feasible method for early diagnosis of metal components. Since Doubov proposed magnetic memory testing, the technology has been widely applied in Russia, Ukraine, Bulgaria, Poland, India, Australia and other countries.

Since the Magnetic Memory Testing technology came out, the scholars have made a lot of research, and have made many remarkable achievements, but it also faces many difficulties and bottlenecks. At present, the research and application of Magnetic Memory Testing technology mainly focus on the micro mechanism research, signal processing and so on.

\section{Research Status of Magnetic Memory Testing Technology}

Microscopic mechanism of Magnetic Memory. Magnetic memory is first found in engineering practice. For its micro mechanism, there are mainly 3 interpretations at present.

First, the explanation based on the magnetostrictive effect and the inverse magnetostrictive effect. According to the Russian scholar Doubov's explain, the mechanism of magnetic memory testing can be expressed as follows. The ferromagnetic metal workpiece, affected by both the magnetic field and work load, deformation and stress concentration will occur within the region of the magnetostrictive properties of the magnetic domain orientation and irreversible reorientation the magnetic state, irreversible changes in work load will not only eliminate the reservation, but also with the maximum stress, which should be interacting with magnetic memory force corresponding to the stress [3].

Second, the explanation based on the principle of energy balance. Ren Jilin of Nanchang Aeronautics and Astronautics University, believes that the formation of the stress concentration area in the ferromagnetic component will gather very high stress energy. According to principle of the actual state of existence must be the minimum energy state, then, to make the total free components can be minimum, rearranging the orientation of displacement in the magneto mechanical effect under the action will cause the internal domain as the domain wall in the geomagnetic field and even irreversible, mainly to increase the magneto elastic performance the form of offset stress can increase, resulting in much higher than the magnetic field strength of the magnetic field strength in a component [4].

Third, the electromagnetic induction theory. Zhong Weichang of Nanjing Gas Turbine Research Institute explains the mechanism of magnetic memory testing according to electromagnetics.

For the mechanism of magnetic memory testing, scholars explain it according to their own opinion, but it has not formed a rigorous and complete theoretical system so far. Compared with the application of magnetic memory testing technology in the engineering, study of magnetic memory mechanism lags behind, the domestic and foreign research results obtained are more concentrated in the processing of magnetic memory and magnetic memory signal detection instrument development and other fields, and in the study of the mechanism of magnetic memory testing is a major breakthrough.

Signal Processing of Magnetic Memory Testing. First, the analysis of magnetic memory testing signals' influencing factors. The researchers find the fact from a lot of magnetic memory testing in practice that if the same workpiece is in different geographical positions, such as the workpiece and 
things are in the southern and Northern direction, or magnetic memory detection instrument is set with different detection parameters set, such as detection probe detection speed, different lift off height, magnetic memory detection of magnetic memory signal acquisition equipment acquisition is not completely consistent. So, the magnetic memory signal is affected by various external factors and randomness, these factors include the earth's magnetic field, detection speed and detection probe lift off, the nature and degree of the influence of different factors on the detection signal is not the same.

In fact, there are many factors affecting the magnetic memory signal in addition to the earth's magnetic field mentioned above [5], such as the external magnetic field, the detection probe and the detection speed provided, etc. while the current research on the relationship between geomagnetic and magnetic memory signal is not systematic, and then the research on this issue still has a larger space for further.

Second, pretreatment of magnetic memory testing signals. Due to the influence of various factors, the magnetic memory testing signal is often mixed with serious noise, and interference analysis, it is particularly necessary for the follow-up, the magnetic memory signal smoothing, noise reduction pretreatment. Because of the significant advantages of wavelet analysis in signal processing, the study of magnetic memory testing signal denoising is mainly focused on the signal denoising based on wavelet analysis.

However, the existing signal preprocessing methods can only eliminate the interference of noise and other factors to a certain extent [6]. Denoising effect remains to be improved. In order to reduce the effect of noise on the detection of magnetic memory signals, a more effective method of magnetic memory signal preprocessing is also needed.

Third, study on criteria for determining the stress concentration zone with magnetic memory testing. The original advantage of magnetic memory testing technique is that it can discover the early damage of the component, and Forecast the Danger area of serious stress concentration in component in order to prevent the occurrence of hazardous accidents. The judgment criterion of the stress concentration area proposed by the Russian scholar

Doubov can be expressed as follows: find the area where the tangential magnetic field strength has maximum value, the normal magnetic field strength change the symbol and has zero points, and then calculate the gradient value $K=\mathrm{d} H_{\mathrm{p}}(y) / \mathrm{d} x$, thus the stress concentration area can be concluded,

However, a large number of engineering practices shows that the above criteria may lead to the omission of the detection or error detection [7]. It can be seen from the corresponding literature that the above criteria are aimed at one or several features extracted from magnetic memory testing signals. The Accuracy and reliability of Criterion have yet to be improved. So, Future development trends is to conduct a more comprehensive and in-depth analysis on Magnetic Memory Signal Feature and to Make the judgment criterion of accuracy and reliability.

Fourth, study on quantitative recognition of stress concentration or disfigurement with Magnetic Memory Testing. The qualitative analysis of the stress concentration zone of the equipment or component can only determine the damage area, but can not meet the requirements of life prediction and evaluation of in-service equipment in engineering practice. So, it needs to conduct a more in-depth analysis on Stress concentration area in order to determine the degree of stress concentration, the type of the defect or the size of the defect.

According to the current domestic and international research situation [8,9], the study of quantitative identification of stress concentration or defect is on the initial stage. The study is mainly focused on feature extraction of magnetic memory signal. There is still a long way to the goal of quantitative identification.

Fifth, research and development of magnetic memory testing instrument. At present, the magnetic memory testing Instrument maker in the world mainly include Russian Power Diagnostics Company, Eddysun (Xiamen) Electronic Company. There are also some other university or company which produce the magnetic memory testing Instrument, such as Tsinghua University, Beijing Institute of Technology, Nanjing University of Aeronautics \& Astronautics and China special equipment inspection and Research Center. While, these instruments can only locate the stress concentration area, 
qualitatively indicate the size of the stress concentration. Besides, missed detection or error detection often occurs in those instruments, and those instruments are unable to quantify the defect.

\section{Trends in the Study of Magnetic Memory Testing}

Strengthen the Research of Microscopic Mechanism. Magnetic memory testing technology is a new cross subject, which involves multi-disciplinary and multi domain knowledge. At present, the research on the microscopic mechanism of magnetic memory testing has been a difficult problem in the academic field. The microscopic mechanism of magnetic memory testing is still not perfect, thus it has not yet formed a relatively complete and rigorous theoretical system. This makes the development of magnetic memory testing technology limited. So, the microscopic mechanism of magnetic memory testing is one of the future research directions in the field of magnetic memory testing.

Quantification of Magnetic Memory Testing. Magnetic memory testing technology is only used as an auxiliary nondestructive testing method at present, using it to detect the suspected stress concentration area or defect parts of the equipment or components [10]. For whether there is a defect, what type of defect it belongs to, and Quantification of defect need to be identified and implemented by using other testing techniques, which greatly limits the application of magnetic memory testing technology in engineering. Therefore, to improve the accuracy and reliability of magnetic memory testing technology, to make the magnetic memory testing technology can be used to identify the defect parameters will have important theoretical significance and practical value. This must be an important development direction of magnetic memory testing.

\section{Conclusions}

As a new nondestructive testing technology, magnetic memory testing technology is the only feasible nondestructive testing method for the early diagnosis of the pipeline. The Scientists have achieved fruitful results in the microscopic mechanism, signal processing, magnetic memory testing criterion, the stress concentration area of magnetic memory testing stress concentration or defect quantitative identification, magnetic memory testing instrument research and development and other aspects of the research and development. With the development of engineering practice, Growing demand for engineering testing will greatly promote and promote the development of magnetic memory testing. Especially the solution to the above hot issues will undoubtedly promote the application of magnetic memory testing technology in petroleum, chemical, aerospace, electric power, transportation and other fields.

\section{Acknowledgements}

This work was financially supported by the Chongqing Postdoctoral Science Foundation.

\section{References}

[1] A. A. Doubov, A study of metal properties using the method of magnetic memory, Metal Sci. Heat Treat. 39(9) (1997) 401-402.

[2] A. A. Doubov, The express technique of welded joints examination with use of metal magnetic memory, NDT\& E Int. 33(6) (2000) 351-362.

[3] A. A. Doubov, Diagnostics of metal and equipment by means of metal magnetic memory, Proceedings of 7th Conference on NDT and International Research Symposium, Shantou China, 1999, 181-187.

[4] J. L. Ren, G. H. Wu, K. Song, Study on the mechanism of metal magnetic memory testing, Nonde Struct. Test. 24(1) (2002) 29-31. In Chinese 
[5] L. M. Li, X. F. Wang, S. L. Huang, The relationship between metal magnetic memory and geomagnetic field, Nonde Struct. Test. 25(8) (2003) 387-389. In Chinese

[6] F. Yi, Z. X. Li, Y. Su, Denoising algorithm for metal magnetic memory signals of oil pipeline based on improved wavelet threshold, Acta Petrolei Sinica, 30(1) (2009) 141-144. In Chinese

[7] Z. F. Liang, Y. N. Wang, W. S. Li, LI Wushen. General character of metal magnetic memory signal in tension experiment, J. Harbin Inst. Technol. 41(5) (2009) In Chinese.

[8] J. Zhang, B. Wang, Recognition of Signals for Stress Concentration Zone in Metal Magnetic Memory Tests, Proc. CSEE, 28(18) (2008) 144-148. In Chinese

[9] J. L. Ren, J. Wang, Z. Z. Fan, New method for metal magnetic memory quantitative analysis, Chin. J. Scient. Instrum. 31(2) (2010) 431-436. In Chinese

[10]X. J. Di, W. S. Li, S. W. Bai, Quantitative Evaluation Study on Metal Magnetic Memory of Welding Cracks, J. Mater. Eng. 7 (2006) 56-60. In Chinese 